\title{
Observações adicionais sobre os pigmentos visuais e filtros amarelos nos olhos de peixes amazônicos.
}

\author{
W. R. A. Muntz (')
}

\section{Resumo}

Em um trabalho anterior (Muntz, 1973), foram apresentados dados sobre os pigmentos visuais de alguns peixes amazônicos, estudados através da mediçāo da absorvância espectral de pequenas áreas de retinas intactas. Em alguns casos, as córneas e lentes foram encontradas, contendo pigmentos filtradores amarelos, para os quais dados de absorvância espectral também foram apresentados. O presente artigo representa uma extensão daquele trabalho, efetuado durante a Fase 5 do cruzeiro Alpha-Helix ao sistema do rio Amazonas em 1977. Os peixes para estudo foram coletados, durante janeiro e fevereiro, de nove locais próximos a Manaus. com ambientes luminosos muito diferentes, e as absorvâncias espectrais de suas retinas, lentes e córneas medidas. Com a exceção de uma espécie de gymnotideo e uma espécie de tetraodontiforme, todos os espécimes foram cichlideos ou characíneos.

\section{MATERIAL E MÉTODOS}

\section{LOCAIS DE COLETAS}

Coletas foram feitas em janeiro de 1977 usando-se isca, rede, ou malhadeira, em nove locais. Dados minuciosos sobre a qualidade espectral e a quantidade total de luz nos locais $A, B, G, I$ e J podem ser encontrados em Muntz (1978). As descrições gerais das características dos rios nesta área podem ser encontradas em Myers (1947) e Sioli (1967).

Local A - Lago Castanho, lago lateral ao rio Solimões. A água é semelhante à do próprio Solimões, sendo que contém menos material em suspensão e é muito mais limpa.

Local $B$ - Foi o canal que liga o lago Castanho ao Solimões. A água era altamente túrbida com material em suspensão, e essencialmente a mesma água do próprio Solimões.

Local G - Foi uma pequena ilha no Solimões, com praias onde se poderia lancear com redes.
Locais $E, F$ e $\mathrm{H}$ - eram pequenos lagos rasos, isolados do sistema do rio principal durante a estação seca. Pela marca da água nas árvores, é evidente que, quando o nível da água estava alto, todos os três lagos ficavam submersos e em contato direto com o Solimões e uns com os outros.

Local I - era no rio Cuieiras, um tributário do rio Negro, próximo de sua junção com o rio principal.

Local $J$ - foi no próprio rio Negro.

Local $K$ - Foi um pequeno igarapé de água clara que entrava no rio Cuieiras.

\section{MEDIDAS DE ABSORVÂNCIA ESPECTRAI}

As absorvâncias espectrais de pequenas áreas de retinas intactas, córneas e lentes foram medidas, usando-se o espectrofotômetro para propósitos especiais descrito em Muntz $(1973,1976)$. Em todos os casos, os peixes eram inicialmente adaptados ao escuro durante, pelo menos, uma hora. As manipulações subseqüentes foram efetuadas usando-se uma luz de segurança fotográfica vermelha, para evitar o descoramento dos pigmentos visuais. Morto o peixe, os seus olhos foram removidos e hemi-sectados, as lentes dissecadas livres e a iris removida da córnea. As retinas eram então libertadas do epitélio do pigmento, e as amostras montadas em lâminas de microscópio em pequenos reservatórios cheios de glicerol (que reduz a dispersão da luz) e cobertas com tampas deslizantes. O espectrofotômetro incorporava uma plataforma de microscópio mecânica que permitia que as amostras fossem movidas de maneira que o ponto de medição retangular de $0,1 \times 0,8 \mathrm{~mm}$ do instrumento caisse sobre a área a ser medida.

(1) - Department of Biology, Stirling University, Scotland. 
A absorvância retinal de uma retina intacta que é separada do epitélio do pigmento é quase inteiramente devida aos pigmentos visuais nos bastonetes (Denton, 1959). Em todos os peixes estudados, a absorvância máxima da retina foi em comprimentos de onda menores que $535 \mathrm{~nm}$. Os pigmentos visuais responsáveis irão, por isso, também absorver maximamente, nesta região espectral, e absorverão muito pouca luz, em comprimentos de onda longos. A absorvância em comprimentos de onda longos, que foi sempre baixa, deve ser devida a substâncias outras que não os pigmentos visuais, ou ao descoramento. Seguindo o procedimento de Denton et. al. (1971), admitiu-se que os pigmentos visuais propriamente ditos absorvem superficialmente em comprimentos de onda maiores que $640 \mathrm{~nm}$, e a absorvância neste comprimento de onda foi adequadamente subtraido daqueles em outros comprimentos de onda de modo a obter-se uma estimativa da densidade do pigmento visual sozinho. Este procedimento supõe que a absorvância da retina sem pigmento visual é expectralmente neutra, o que é pelo menos aproximadamente verdade (Denton, 1959).

\section{Resultados}

Os resultados estão resumidos na Tabela I e Figura 2, e um exemplo do tipo de dados que são obtidos, usando-se a presente técnica é dado na Figura 1. Na Tabela, os pigmentos visuais são caracterizados pelos seus $\lambda 50$, que é o ponto no lado do comprimento de onda longa da curva de absorção retinal em que a

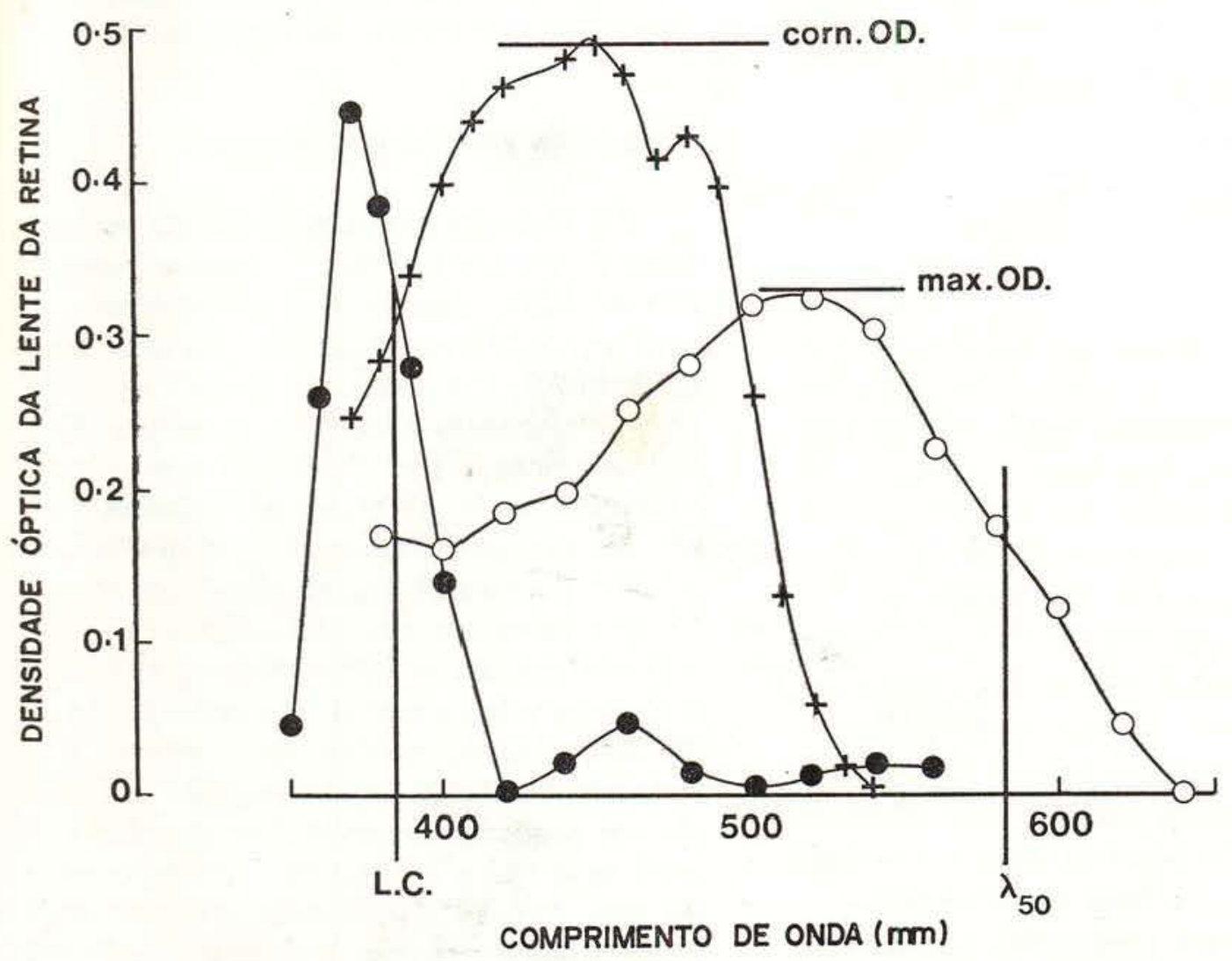

Fig. 1 - Características espectrais das lentes, córnea e retina de Colomesus asellus. Círculos cheios, lente; círculos vazios, retina; cruzes, córnea. As medições dadas na Tabela I são indicadas como se segue: L.C., ponto de interrupção de 0,3 unidades de $\log$ da lente; $\lambda 50$, comprimento de onda no qual a absorção retinal cai para $50 \%$ de seu valor máximo, max. O.D., densidade ótica máxima da retina; corn. O.D., densidade ótica da córnea. As medições das lentes e retina foram feitas em um exemplar adaptado ao escuro, as mediçōes corneais em exemplares adaptados à luz. 
densidade ótica declinou para $50 \%$ de seu valor máximo, uma vez que isto é mais fácil de estimar com precisão do que o comprimento de onda de absorção máxima ( $\lambda \max$ ). A densidade ótica da retina em seu max é também fornecida. Como em Muntz (1972), a cor das lentes é dada como a unidade de log 0,3 do ponto de interrupção, que é o comprimento de onda, no qual as absorções das lentes se elevam a 0,3 unidade log acima de sua absorção em $550 \mathrm{~nm}$. As densidades corneais são dadas como a diferença entre as densidades a $450 \mathrm{~nm}$ e $550 \mathrm{~nm}$. Todas estas medições estão indicadas na Figura 1.

\section{LENTES E CÓRNEAS AMARELAS}

Córneas amarelas foram comuns nos cichlídeos (ver Tabela 1). O pigmento amarelo era freqüentemente restrito à parte dorsal da córnea e mesmo quando não restrito a ela, era sempre mais denso nesta área. Córneas pigmentadas em todos os casos absorveram maximamente em torno de $450 \mathrm{~nm}$ e tornava-se transparente novamente em comprimentos de onda mais curtos, e medições minuciosas mostraram claramente que alguma forma da carotenóide estava envolvida (Figura 2). Onde mais de um exemplar de uma dada espécie foi estudado, houve sempre alguma variabilidade na espessura da pigmentação encontrada. Estes resultados confirmam e ampliam os registrados em Muntz (1973).

No registro anterior, córneas amarelas não foram encontradas em characíneos. No presente estudo, no entanto, pigmento amarelo foi observado nas córneas de duas espécies de characíneos, Chalceus macrolepidotus e uma espécie de Mylossoma (a última não está incluída nem na Tabela I nem na Figura 2, porque foi impossível dissecar a retina inteira), Em ambos os casos, o pigmento era restrito a uma mancha em forma decrescente na córnea dorsal. As características espectrais do pigmento de Mylossoma são apresentadas na $\mathrm{Fi}$ gura 3. Parece provável que a faixa de absorção em torno de $450 \mathrm{~nm}$ é devida a um carotenóide semelhante ao encontrado nos cichlídeos, e que outros pigmentos são responsá- veis pela absorção em comprimentos de ondas mais curtos. Embora córneas amarelas fossem encontradas nestes dois characíneos, outras observações feitas durante o presente estudo confirmam que elas são incomuns. Somente sete characíneos contribuem para a Tabea I e Figura 2, porque, na maioria dos casos, foi muito difícil dissecar as retinas inteiras, mas em um grande número de outras espécies as córneas e lentes eram olhadas e encontradas sem cor.

Uma córnea amarela foi também encontrada em espécimes adaptados à luz do baiacu, Colomesus asellus (Fig. 1). Em espécimes adaptados ao escuro, contudo, a córnea era sem cor, sendo espectralmente neutra e com uma densidade ótica máxima de 0,05 . Sob microscópio, pode ser visto que as margens dorsal e ventral da córnea eram contornadas por cromatóforos com processos estendendo-se sobre a córnea, e que o pigmento amarelo migrava para baixo destes processos, na luz. A presença de tais córneas amarelas "oclusivas" foi registrada anteriormente por Orlov \& Gamburtzeva (1976) para Hexagrammideos, e por Appleby \& Muntz (1979) para três espécies de baiacus de águas salobras do Sudeste da Ásia.

Lentes amarelas ocorreram em muitos cichlídeos, e em um characíneo (Chalceus macrolepidotus). Na Tabela I, elas são caracterizadas pela unidade $0,3 \log$ do ponto de interrupção, como em Muntz $(1972,1973)$. Contudo, em alguns casos, a quantidade de pigmentos nas lentes até $380 \mathrm{~nm}$. Alguns exemplos são apresentados nas Figuras 1 e 4, e os resultados para estas espécies estão resumidos na Tabela II, que também apresenta a aparência visual das lentes. Em todos os casos, as curvas de absorvância foram estreitas, com máximos bem definidos em, ou ao redor de $400 \mathrm{~nm}$. Parece provável que, em espécies com lentes fortemente amarelas, pigmentos semelhantes estão envolvidos em concentrações mais elevadas. A absorvância espectral de um pequeno fragmento de uma lente de Cichlosoma festivum foi por isso medida. Os resultados, que estão apresentados na Figura 4, estão de acordo com esta suposição. Os diferentes pontos de interrupção das lentes das várias espécies, 
TABELA I - Resumo dos resultados

\begin{tabular}{|c|c|c|c|c|c|}
\hline \multirow{2}{*}{ Espécie } & \multirow{2}{*}{$\begin{array}{l}\text { Local de } \\
\text { coleta }\end{array}$} & \multicolumn{2}{|c|}{ Retina } & \multirow{2}{*}{$\begin{array}{l}\text { Interrupção } \\
\text { da lente } \\
\text { (nm) }\end{array}$} & \multirow{2}{*}{$\begin{array}{c}\text { Pigmento corneal } \\
\text { densidade }\end{array}$} \\
\hline & & $\begin{array}{l}\lambda 50 \\
(\mathrm{~nm})\end{array}$ & $\begin{array}{l}\max \\
\text { O.D. }\end{array}$ & & \\
\hline $\begin{array}{l}\text { Cichlídeos da Região } \\
\text { do Solimões }\end{array}$ & & & & & \\
\hline $\begin{array}{l}\text { Cichlasoma } \\
\text { temporale }\end{array}$ & A & 548 & 0,36 & 438 & $\begin{array}{l}0,25 \text { no centro, } \\
\text { mais escuro dorsalmente }\end{array}$ \\
\hline Cichlasoma sp. & A & 558 & 0.55 & 472 & $\begin{array}{l}0,05 \text { no centro, } \\
0,36 \text { no bordo dorsal }\end{array}$ \\
\hline Cichlasoma sp. & A & 568 & 0,56 & 422 & 0 no centro, 0,47 no bordo dorsal \\
\hline Cichlasoma severum & A & 548 & 0,38 & 438 & $\begin{array}{l}0,5 \text { no centro, } 0,90 \text { no } \\
\text { bordo dorsal }\end{array}$ \\
\hline Crenicichla lugubris & A & veja & texto & - & $\begin{array}{l}0,18 \text { no centro, pequena } \\
\text { mancha escura acima }\end{array}$ \\
\hline $\begin{array}{l}\text { Uaru } \\
\text { amphiacanthoides }\end{array}$ & A & 558 & 0.64 & 426 & - \\
\hline $\begin{array}{l}\text { Uaru } \\
\text { amphiacanthoides }\end{array}$ & A & 554 & 0,87 & 426 & - \\
\hline Acarichthys heckelli & A & 576 & 0,30 & - & - \\
\hline Geophagus acuticeps & A & 562 & 0,58 & - & - \\
\hline Geophagus acuticeps & A & 555 & 0,85 & $400^{\prime}$ & - \\
\hline Apistogramma sp. & A & 554 & 0,20 & - & - \\
\hline $\begin{array}{l}\text { Chaetobranchopsis } \\
\text { opercularis }\end{array}$ & $\mathrm{F}$ & 578 & 0,34 & 442 & - \\
\hline $\begin{array}{l}\text { Chaetobranchopsis } \\
\text { opercularis }\end{array}$ & $\mathrm{F}$ & 584 & 0,52 & 448 & 0,15 no centro, cor uniforme \\
\hline Cichlasoma severum & F & 562 & 0,46 & 426 & $\begin{array}{l}0,15 \text { no centro, margem dorsal } \\
\text { não medida }\end{array}$ \\
\hline Pterophylum scalare & F & 580 & 0.47 & 424 & - \\
\hline Cichlasoma festivum & E & 566 & 0,37 & 440 & $\begin{array}{l}0,15 \text { no centro, } 0,7 \text { no bordo } \\
\text { dorsal }\end{array}$ \\
\hline
\end{tabular}




\begin{tabular}{|c|c|c|c|c|c|}
\hline \multirow{2}{*}{ Espécie } & \multirow{2}{*}{$\begin{array}{l}\text { Local de } \\
\text { coleta }\end{array}$} & \multicolumn{2}{|c|}{ Retina } & \multirow{2}{*}{$\begin{array}{l}\text { Interrupção } \\
\text { da lente } \\
\text { (nm) }\end{array}$} & \multirow{2}{*}{$\begin{array}{l}\text { Pigmento corneal } \\
\text { densidade }\end{array}$} \\
\hline & & $\begin{array}{r}\lambda 50 \\
(\mathrm{~nm})\end{array}$ & $\begin{array}{l}\max . \\
\text { O.D. }\end{array}$ & & \\
\hline Cichla ocellaris & $\mathrm{H}$ & 582 & 0,46 & - & $\begin{array}{l}\text { quarto dorsal da córnea amarelo } \\
\text { mas não medido }\end{array}$ \\
\hline $\begin{array}{l}\text { Geophayus } \\
\text { surinamensis }\end{array}$ & $\mathrm{H}$ & 580 & 0,20 & - & - \\
\hline \multicolumn{6}{|l|}{$\begin{array}{l}\text { Characíneos da Região } \\
\text { do Solimões }\end{array}$} \\
\hline $\begin{array}{l}\text { Chalceus } \\
\text { macrolepidotus }\end{array}$ & A & 578 & 0,39 & 430 & $\begin{array}{l}0,2 \text { no centro, acima de } 0,5 \\
\text { no bordo dorsal }\end{array}$ \\
\hline Roeboides sp. & A & 578 & 0,40 & - & - \\
\hline $\begin{array}{l}\text { Chalceus } \\
\text { macrolepidotus }\end{array}$ & $\mathrm{H}$ & 584 & 0,57 & 434 & $\begin{array}{l}0,22 \text { no centro, acima de } 0,6 \\
\text { no bordo dorsal }\end{array}$ \\
\hline Roeboides sp. & B & 572 & 0,36 & - & - \\
\hline $\begin{array}{l}\text { Semelcarinata } \\
\text { isognatha }\end{array}$ & G & 586 & 0,38 & - & - \\
\hline Curimatus sp. & G & 588 & 0,42 & - & - \\
\hline $\begin{array}{l}\text { Curimatus cf. } \\
\text { hypostomus }\end{array}$ & G & 590 & 0,23 & - & - \\
\hline \multicolumn{6}{|l|}{$\begin{array}{l}\text { Outros peixes da } \\
\text { Região do Solimões }\end{array}$} \\
\hline Eigenmannia $\mathrm{sp}$. & G & 588 & 0.46 & - & - \\
\hline Colomesus aselius & A & 576 & 0,15 & 405 & $\begin{array}{l}\text { Oclusivel, atingindo } 0,78 \\
\text { quando coberta }\end{array}$ \\
\hline \multicolumn{6}{|l|}{$\begin{array}{l}\text { Cichlídeos da Região } \\
\text { do rio Negro }\end{array}$} \\
\hline Geophagus sp & $\mathrm{J}$ & 586 & 0,35 & - & - \\
\hline Geophagus sp & $J$ & 580 & 0,33 & - & - \\
\hline Geophagus daemon & $\mathrm{J}$ & 552 & 0,66 & - & - \\
\hline Cichlasoma festivum & $\mathrm{J}$ & 556 & 0,27 & 430 & $\begin{array}{l}0,13 \text { no centro, } 0,34 \text { no bordo } \\
\text { dorsal }\end{array}$ \\
\hline
\end{tabular}

Observações... 


\begin{tabular}{|c|c|c|c|c|c|}
\hline \multirow{2}{*}{ Espécie } & \multirow{2}{*}{$\begin{array}{l}\text { Local de } \\
\text { coleta }\end{array}$} & \multicolumn{2}{|c|}{ Retina } & \multirow{2}{*}{$\begin{array}{l}\text { Interrupção } \\
\text { da lente } \\
(\mathrm{nm})\end{array}$} & \multirow{2}{*}{$\begin{array}{l}\text { Pigmento corneal } \\
\text { densidade }\end{array}$} \\
\hline & & $\begin{array}{l}\lambda 50 \\
(\mathrm{~nm})\end{array}$ & $\begin{array}{l}\max \\
\text { O.D. }\end{array}$ & & \\
\hline Cichla temensis & J & 570 & 0,22 & 405 & $\begin{array}{l}0,11 \text { no centro, } 0,56 \text { no } \\
\text { bordo dorsal }\end{array}$ \\
\hline Acaronia nassa & 1 & 552 & 0,90 & - & - \\
\hline Acaronia nassa & 1 & 556 & 0,30 & - & - \\
\hline Acaricthys heckelli & 1 & 558 & 0,47 & - & 一 \\
\hline Cichlasoma severum & K & 552 & 0,24 & - & $\begin{array}{l}\text { Faixa amarela no bordo } \\
\text { dorsal, não medida }\end{array}$ \\
\hline Cichlasoma severum & K & 550 & 0,78 & 436 & $\begin{array}{l}0,05 \text { no centro, } 0,65 \text { no } \\
\text { bordo dorsal }\end{array}$ \\
\hline $\begin{array}{l}\text { Aequidens } \\
\text { duopunctatum }\end{array}$ & K & 548 & 0,61 & 432 & $\begin{array}{l}0 \text { no centro, } 1,0 \text { no bordo } \\
\text { dorsal }\end{array}$ \\
\hline $\begin{array}{l}\text { Aequidens } \\
\text { duopunctatum }\end{array}$ & K & 550 & 0,67 & 436 & $\begin{array}{l}0,08 \text { no centro, } 0,8 \text { no bordo } \\
\text { dorsal }\end{array}$ \\
\hline $\begin{array}{l}\text { Outros peixes da } \\
\text { Regiāo do rio Negro }\end{array}$ & & & & & \\
\hline Colomesus asellus & $J$ & 583 & 0,33 & 385 & $\begin{array}{l}\text { Oclusível, atingindo } 0,98 \\
\text { quando coberta }\end{array}$ \\
\hline
\end{tabular}

(1) - Com esta interrupçăo, a lente não tinha cor visivel.

por isso, provavelmente refletem diferentes concentrações de pigmentos que são semeIhantes, em todas as espécies. Pigmentos muito semelhantes foram também encontrados nas lentes do teleósteo mesopelágico Scopelachus analis, da lula mesopelágica Histioteuthis meleagroteuthis (ver Muntz, 1976), esquilos (Cooper \& Robson, 1969a) e primatas (Cooper \& Robson, 1969b).

Em uma espécie de cichlídeo, Crenicichla lugubris, a própria retina era de cor amarela forte, absorvendo maximamente ao redor de $470 \mathrm{~nm}$ e com uma densidade ótica de 0,48 neste comprimento de onda. Isto concorda com os achados em outra espécie de Crenicichla, C. lenticulata, registrado em Muntz (1973).

\section{PIGMENTOS VISUAIS}

Os cichlídeos amazônicos usualmente possuem dois pigmentos visuais extraíveis na retina, um baseado na vitamina $A$, e absorvendo maximamente em $500 \mathrm{~nm}$, e o outro baseado na Vitamina $A_{2}$ e absorvendo maximamente em 522 nm (Schwanzara, 1967). A absorvância espectral da retina intacta de um cichlídeo depende da proporção relativa dos dois pigmentos contidos nela. Se somente o primeiro pigmento está presente, o $\lambda$ max será em 500 nm e o $\lambda 50$ ao redor de $550 \mathrm{~nm}$; se somente o último, o $\lambda$ max será em $522 \mathrm{~nm}$ e o $\lambda 50$ ao redor de $585 \mathrm{~nm}$; as proporções intermediárias dos dois pigmentos darão valores intermediá- 


\section{ÁREA DO SOLIMÖES}

\section{Cichlídeos}

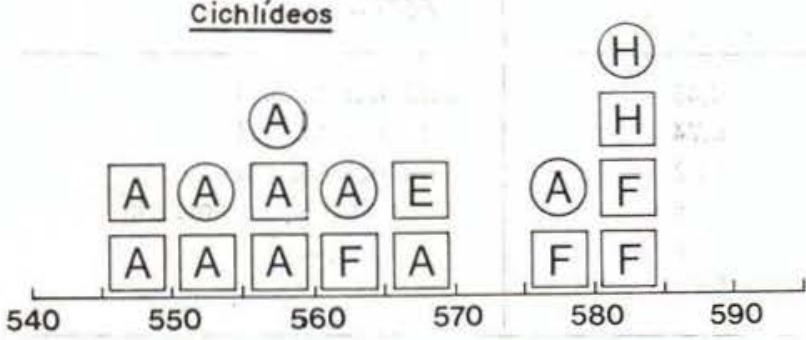

\section{Carachins}

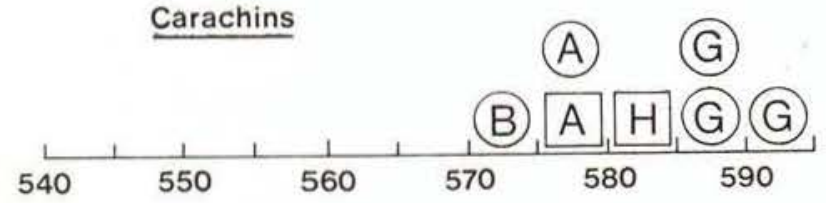

\section{ÁREA DO RIO NEGRO}

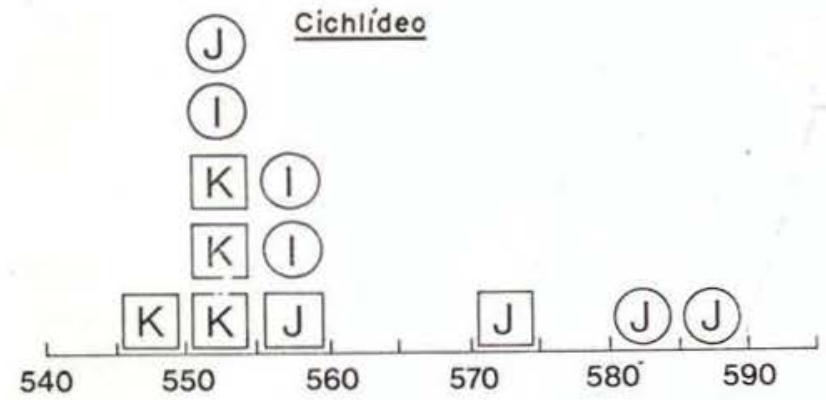

$$
\lambda_{50}(\mathrm{~nm})
$$

Fig. 2 - Resumo dos resultados sobre retinas. O eixo horizontal mostra o comprimento de onda do $\lambda 50$, e cada símbolo representa um exemplar diferente Os símbolos quadrados mostram exemplares com córneas ou lentes amarelas, os símbolos circulares peixes sem cor visível nestas estruturas. As letras dentro dos símbolos referem-se aos locais de coleta.

rios para o $\lambda \max$ e $\lambda 50$. Da Tabela I e Figura 2 , pode ser visto que as espécies de cichlídeos, para ambas as áreas, do Solimões e rio Negro, cobrem a amplitude total possível de valores de $\lambda 50$, sugerindo que os dois pigmentos podem ocorrer em qualquer proporção relativa.

Characíneos da região Amazônica também geralmente possuem dois pigmentos visuais, um pigmento baseado em $A_{1}$ com $\lambda$ max em $503 \mathrm{~nm}$ é seu $\lambda 50$ por volta de $555 \mathrm{~nm}$, e um pigmento baseado em $A_{2}$ com seu $\lambda \max$ em $527 \mathrm{~nm}$ e seu $\lambda 50$, ao redor de $590 \mathrm{~nm}$ (Schwanzara, 1967). Ao contrário dos cichlídeos, os resultados dos characíneos indicam que todos eles possuem proporções comparativamente altas de pigmentos baseados em $\mathrm{A}_{2}$ na retina, uma vez que o menor $\lambda 50$ registrado foi de $572 \mathrm{~nm}$.

Estes resultados estão também em concordância com os de Muntz (1973). Naquele estudo, os valores de $\lambda 50$ das retinas de 11 cichlídeos variaram de $550 \mathrm{~nm}$ até $585 \mathrm{~nm}$, novamente representando todas as proporções possíveis dos dois pigmentos, enquanto que os valores de $\lambda 50$ de 7 characíneos variaram de $565 \mathrm{~nm}$ até $590 \mathrm{~nm}$, mostrando que houve uma predominância de pigmentos baseados em $A_{2}$ em todos os casos. A Figura 5 mostra os resultados combinados para cichlídeos $\mathrm{e}$ characíneos dos estudos atual e anterior. No conjunto, 43 cichlídeos e 14 characíneos contribuiram para esta figura, e um teste $U$ de Mann-Whitney (Siegel, 1956) sobre os valores originais de $\lambda 50$ mostra que a diferença entre os dois grupos é altamente significante $(P$, two tailed $=0,00028$ ) .

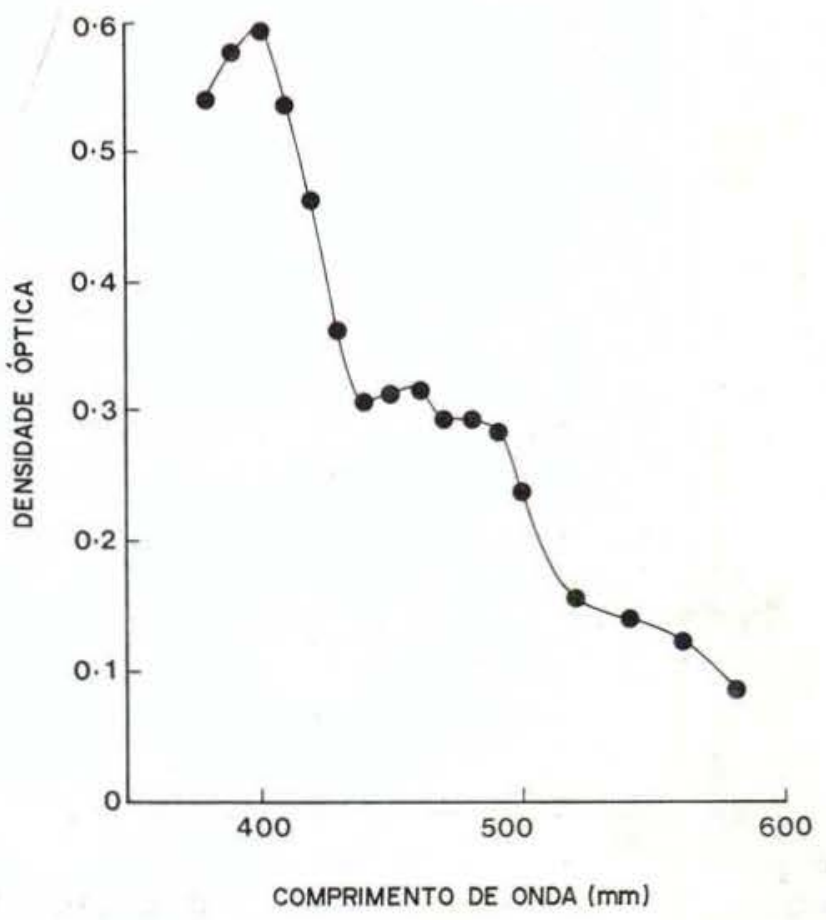

Fig. 3 - Absorvância espectral da área pigmentada de uma córnea de Mylossoma sp. 
TABELA II - Caracteristicas das lentes, em casos em que um completo espectrum de absorção foi obtido

\begin{tabular}{|c|c|c|c|}
\hline Espécies & $\begin{array}{c}\text { interrupção a } 0,3 \\
(\mathrm{~nm})\end{array}$ & $\begin{array}{l}\max . \text { O.D. } \\
\text { Ientes inteiras }\end{array}$ & Aparência \\
\hline Colomesus ascellus & 385 & 0,45 & sem cor visível \\
\hline Geophagus anticeps & 400 & 0,74 & sem cor visivel \\
\hline Cichla temensis & 405 & 0,92 & sem cor visível \\
\hline Uaru amphiacanthoides & 420 & 0,81 & leve coloração amarela \\
\hline Uaru amphiacanthoides & 426 & 0,94 & amarela pálido \\
\hline Chalceus macrolepidotus & 434 & 1,05 & amarela \\
\hline
\end{tabular}

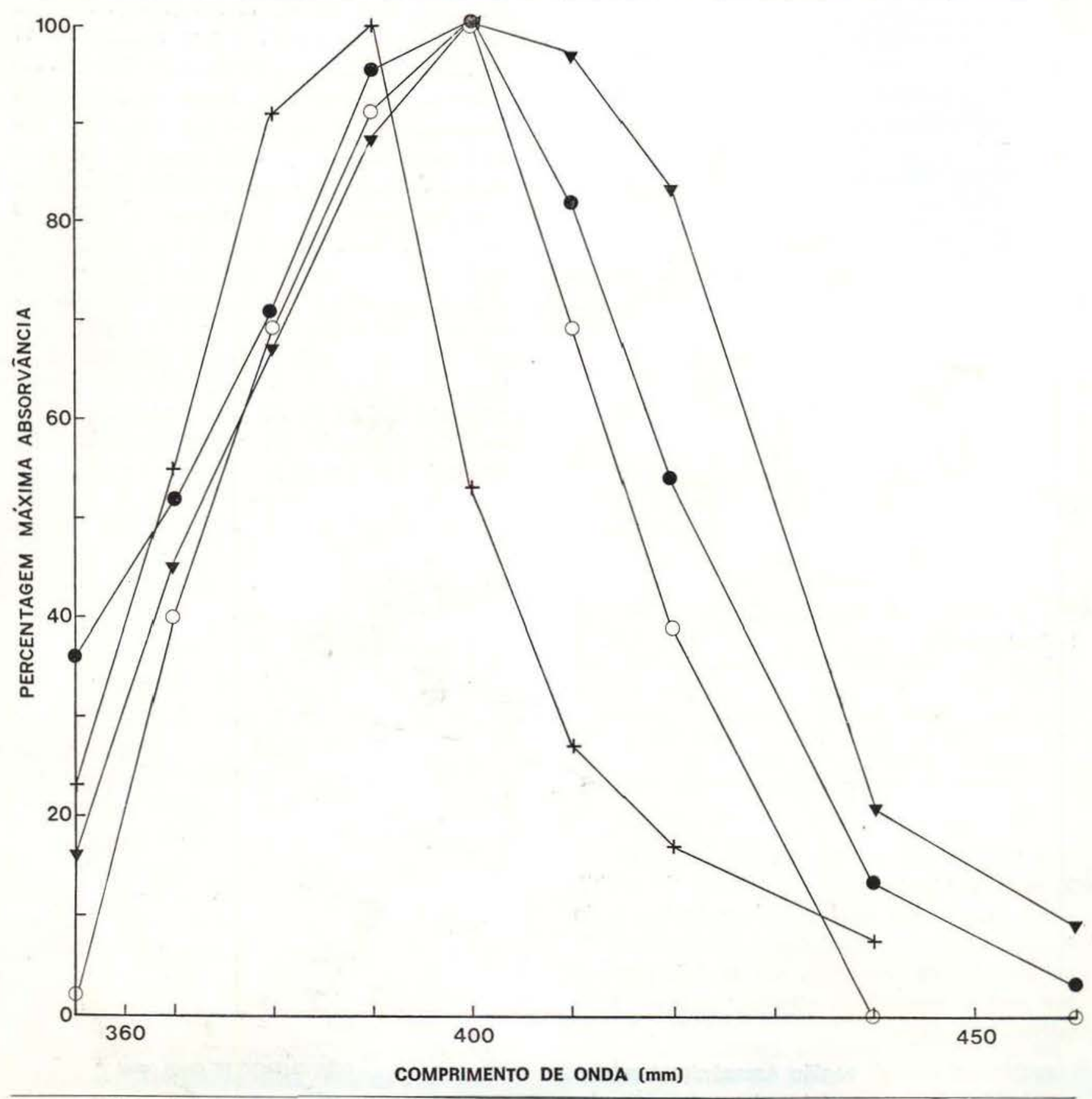

Fig. 4 - Absorvâncias de comprimentos de ondas curtas de lentes selecionadas, Triângulos cheios, Chalceus macrolepidotus; círculos cheios, Uaru amphiacanthoides; círculos vazios, fragmento de lente de Cichlasoma festivum; cruzes, Geophagus anticeps. 


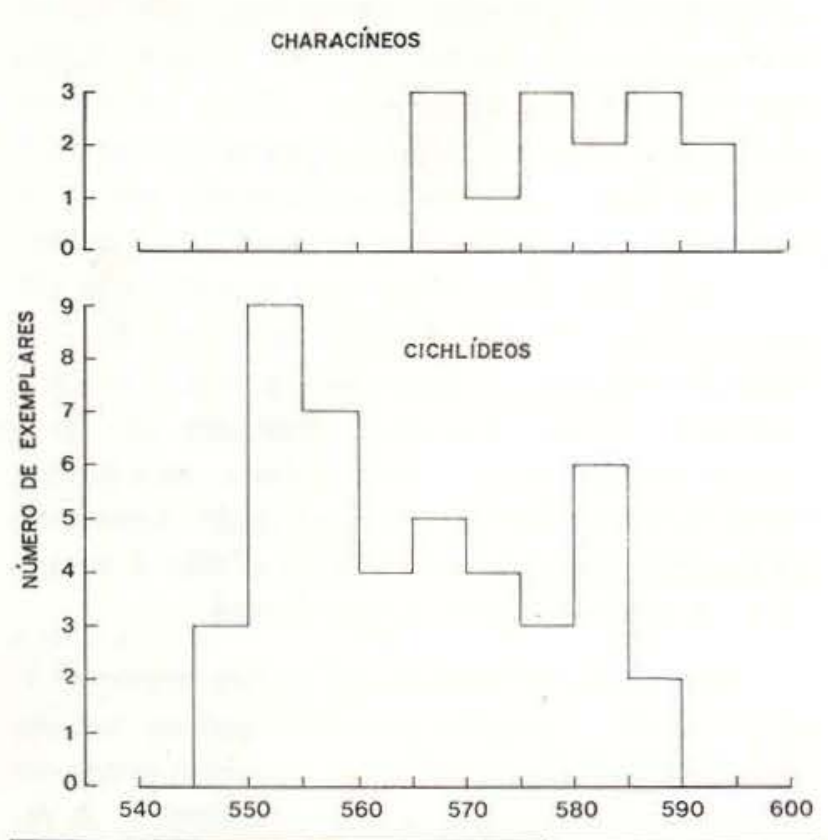

Fig. 5 - Resumo dos resutados sohre as retinas de todos os cichlídeos e characíneos do atual estudo e de Muntz (1973).

Embora a posição dos $\lambda 50$ fosse muito variável, o exame da Figura 2 mostra que houve uma tendência para os peixes habitando águas mais claras terem curvas de absorção retinal lccalizadas em comprimentos de onda mais curtos. Por exemplo, na região do Solimões, o $\lambda 50$ dos cichlídeos do local A (lago Castanho) estava geralmente situado em comprimentos de onda mais curtos que os dos cichlídeos dos locais $\mathrm{E}, \mathrm{F}$ e $\mathrm{H}$ (pequenos lagos rasos), e um teste $U$ de Mann-Whitney mostra que esta diferença é significativa $(P<0,001)$. Enquanto os locais $\mathrm{E}, \mathrm{F}$ e $\mathrm{H}$ eram muito rasos para permitir que medições de luz fossem feitas, a água era certamente menos clara que no local $A$. $\mathrm{Na}$ região do rio Negro, os cichlídeos do local $\mathrm{K}$ (um pequeno igarapé de água clara) tinham retinas que, no total, absorviam luz em comprimentos de onda mais curtos que aqueles cichlídeos do rio Cuieiras e do próprio rio Negro. Aqui, novamente, enquanto não foi possível fazer medições de luz no local $\mathrm{K}$, a água era certamente menos preta que nos locais I e J. Neste caso, o número de espécimes obtidos foi muito pequeno para permitir que qualquer com. paração estatística fosse feita, como foi também o caso para os characíneos.
A Figura 2, também, mostra que não houve diferenças evidentes nos pigmentos visuais de espécies, possuindo ou não possuindo lentes e córneas amarelas.

\section{DISCUSSÃo}

Uma conseqüência inevitável, ao possuir filtros amarelos, é uma perda de sensibilidade. uma vez que tais filtros absorverão muitas quanta de luz que caso contrário teriam atingido a retina. Filtros amarelos são especialmente comuns entre os cichlídeos. Estes peixes não possuem tapeta, seus olhos contêm muitos cones bem desenvolvidos em padröes regulares de mosaico, e testes de comportamento tem mostrado que sua acuridade visual é boa (Weler, 1966). Eles são usualmente de hábitos diurnos (Lowe-McConnel, 1969). Parece assim que os olhos dos cichlídeos são adaptados para visão minuciosa sob niveis de iluminação alta, mas podem ter relativamente baixa sensibilidade e não ser tão adaptados para condições onde os níveis de luz são baixos. É possível que os cichlideos evitem as conseqüências de possuirem uma sensibilidade visual relativamente baixa de duas maneiras. Primeiramente, eles foram encontrados em todos os locais de amostragem exceto no Solimões e no canal de conexão entre o Solimões e o lago Castanho. Durante o período do estudo, estas duas áreas eram caracterizadas por possuirem uma grande quantidade de material em suspensão na água, com conseqüentemente uma extinção da luz muito rápida com o aumento da profundidade: nesta circunstância, eles representaram ambientes onde a visão seria muito mais difícil que em qualquer outros dos locais estudados (Muntz, 1978). Characíneos, gymnotídeos, e bagres foram, contudo, comuns tanto no Solimões quanto no canal. O primeiro grupo caracteristicamente possui tapeta, que aumentará sua sensibilidade, enquanto que os últimos dois grupos possuem olhos pequenos $\mathrm{e}$ confiam grandemente nos outros sistemas sensoriais, tais como as linhas laterais e eletrorrecepção. Filtros amarelos ocorrem só rara- 
mente nestes grupos, e os poucos characíneos que tinham filtros amarelos foram encontrados em lagos, não no canal ou no Solimões. De fato, o único peixe que possui filtros amarelos que foi encontrado no Solimões e no canal foi o baiacu de água doce, e, neste caso, a córnea amarela tornava-se sem cor sob condições de pouca luz.

A segunda maneira, pela qual os cichlideos podem evitar as conseqüências de sensibilidade relativamente baixa é ficando inativo à noite, imóvel, e geralmente encostado contra o fundo ou algum outro objeto submerso (Lowe-McConnell, 1969). Eles conseqüentemente não têm que competir sob condições para as quais eles não estão bem adaptados. Como foi salientado por Muntz (1973), outros grupos nos quais os filtros amarelos são especialmente comuns, tais como os (Labrídeos) (Wrasses) e os peixes papagaios, também mostram métodos especializados de passar a noite em um estado inativo.

Os resultados sobre as retinas mostram que há uma tendência, mesmo não sendo muito bem definida, para que os peixes que habitam águas relativamente claras possuirem curvas de absorção retinal localizadas em comprimentos de onda relativamente curtos. Brid ges (1965) registrou uma diferença semelhante nos pigmentos visuais de duas populações de Notemegonius habitando ambientes claros e túrbidos. Uma vez que a quantidade de luz de comprimento de onda curto é maior nas águas claras que em águas túrbidas ou escuras, esta tendência está na direção correta para aumentar a sensibilidade. Contudo como Dartnall (1974) e outros enfatizaram çue o ganho em sensibilidade resultante de tal mudança de pigmento visual será muito pequenc. Pode ser que as absorções retinais medidas, neste estudo, sejam somente indiretamente relevantes para o problema, uma vez que somente fornece informações sobre o pigmento visual dos bastões. Tem sido mostrado que quando a proporção de pigmentos baseados em $A_{1}$ e $A_{2}$ muda nos bastões também muda nos cones (Loew \& Dartnall, 1976); e desde que a resultante mudança em sensibilidade é muito maior nos cones que nos bastões, par- ticularmente naqueles cones que são sensiveis em comprimentos de onda longos (Liebman, 1972; Loew \& Dartnall, 1976), pode ser aqui que a significância adaptativa das proporções variáveis de pigmento baseado em $A_{1}$ e $A_{2}$ permaneça. Se for este o caso, a descoberta de que não existe diferença consistente entre as curvas de absorvância espectral das retinas de peixes que possuem e não possuem lentes e córneas amarelas, torna-se um problema menor desde que embora tais filtros marcadamente atinjam a qualidade espectral da luz que atinge a retina, este efeito é restrito a comprimentos de ondas curtas.

Em combinação com os dados anteriores, os resultados também mostram que as retinas de characíneos absorvem em comprimentos de onda mais longos que os dos cichlídeos. A razão disto é desconhecida, embora possa estar relacionada, em parte, ao fato de que o raio de ação dos characíneos inclui águas mais túrbidas.

\section{SUMMARY}

In a previous paper (Muntz 1973) data were presented on the visual pigments of a number of Amazonian fishes, studied by measuring the spectral absorbance of small areas of intact retinas. In some cases the corneas and lenses were found to contain yellow filtering pigments, for which spectral absorbance data were also presented. The present paper represents an extension of that work, undertaken during Phase 5 of Alpha-Helix cruise to the Amazon River system in 1977. Fishes for study were collected durıng January and February from nine sites near Manaus, with very different light environments, and the spectral absorbances of their retinas, lenses, and corneas measured. With the exception of one gymnotid and one tetraodontiform species, all the specimens were cichlids or carachins.

\section{REFERÊNCIAS BIBLIOGRÁFICAS}

Appieby, SJ. \& MUNTZ, W.R.A.

1979 - Occlusable yellow corneas in Tetraodontidae. J. Exp. Bio. 83: 249-259.

BRIDGES, C.D.B.

1005 - Variability and relationships of fish visual pigments. Vision Res. 5: 239-251.

COOPER, G.F. \& ROBSON, J.G,

1969a- The yellow colour of the lens of the grey squirrel (Sciurus carolinensis leucotis). J. Physiol. 203. 403-410. 
$1969 \mathrm{~b}$ - The yellow colour of the lens of man and other primates. J. Physiol. 203: 411-417.

DARTNALL, H.J.A.

1974 - Visual pigments and photic environments. In: Vision in Fishes, ed M.A. Ali, NATO Advanced Studies Institutes Series, Plenum Press.

DENTON, B.J.

1959 - The contributions of the oriented photosen sitive and other melecules to the absorption of whole retina. Proc. Roy. Soc. B. 150: 78-94.

Denton, B.J.; MINTZ, W.R.A.; NORThMORE, D.P.M.

1971 - The distribution of visual pigment within the retina in two teleosts. J. mar. biol. Assn. U.K. 51: 905-915.

LeIbMan, P.A.

1972 - Microspectrophotometry of photoreceptors. In: Handbook of Sensory Physiology, vol. VII/I, Photochemistry of Vision, (ed H.J.A. Dartnall). Springer-Verlag, Berlin.

Loew, E.R. \& DarTnall, H.J.A.

1976 - Vitamin $A_{1} / A_{2}$-based visual pigment mistures in cones of the rudd. Vision Res. 16: 891-896.

LOWE-MCCONNEL, R.H.

1969 - The cichlid fishes of Guyana, S. America, with notes on their ecology and breeding behaviour. J. Zool. Limm. Soc. 48: 255302.

MUNTZ, W.R.A.

1972 - Inert reflecting and absorbing pigments. In: Handbook of Sensory Physiology, Vol. VII/I. Photochemistry of Vision 'ed. H.J.A. Dartnall). Springer-Verlag, Berlin.
1973 - Yellow filters and the absorption of light by the visual pigments of some Amazonian fishes. Vision Res. 13: 2235-2254.

1976a- On yellow lenses in mesopelagic animals. J. mar. biol. Assn. U.K. 56: 963-976.

$1976 \mathrm{~b}$ - Visual pigments of cichlid fishes from Malawi. Vision Res. 16: 897-903.

1978 - The penetration of light into Amazonian waters. Acta Amazonica, 8: 613-619.

MYERS, G.S.

1947 - The Amazon and its fishes. Part I. The river. Aquar. J. 18: 4-9.

Orlov, O.Y, \& Gamburtzeva, A.G.

1976 - Changeable colouration of cornea in the fish Hexagrammos octogrammus. Nature 263: $405-407$.

SCHWANZARA, S.A.

1967 - The visual pigments of freshwater fishes Vision Res. 7: 121-148.

SIEgEL, $\mathrm{S}$.

1956 - Nonparametric Statistics. McGraw-Hill.

SIOLI, $\mathrm{H}$

1967 - Studies in Amazonian waters. Atlas do Sim. posio sobre a Biota Amazonica, 3: 9-50.

WEILER, I.V.

1966 - Restoration of visual acinty after optic nerve section in Astronotus ocellatus. Exp. Neurol. 15: 377-386.

(Aceito para publicação em $24 / 10 / 80$ ) 